\title{
Simvastatin improves learning and memory in control but not in olfactory bulbectomized rats
}

\author{
Tessa N. Douma • Yuliya Borre • \\ Hendrikus Hendriksen • Berend Olivier • \\ Ronald S. Oosting
}

Received: 22 July 2010 / Accepted: 21 February 2011 /Published online: 8 March 2011

(C) The Author(s) 2011. This article is published with open access at Springerlink.com

\begin{abstract}
Rationale Olfactory bulbectomy (OBX) in a laboratory rodent leads to numerous behavioral deficits and involves cognitive and motor changes that are used to model major depression, but may also be a valuable tool in the study of neurodegenerative disorders like Alzheimer's disease.

Objectives This experiment evaluated the effects of simvastatin, a cholesterol-lowering drug with putative neuroprotective properties, on OBX-induced behavioral changes.

Results Chronic administration of simvastatin, starting $48 \mathrm{~h}$ after surgery, did not have any behavioral effect in OBX rats, as tested in open field, passive avoidance and objectrecognition paradigms. In control rats, simvastatin treatment resulted in an improved performance in both the passive avoidance and the object-in-place task.

Conclusion In the present study, simvastatin treatment enhanced cognition in intact rats, but had no effect in OBX rats. These results are in line with the idea that statins may attenuate (early) age-associated cognitive decline in humans.
\end{abstract}

Keywords Olfactory bulbectomy · Simvastatin .

Alzheimer's disease · Passive avoidance - Open field .

Object-place recognition

T. N. Douma $(\bowtie) \cdot$ Y. Borre $\cdot$ H. Hendriksen $\cdot$ B. Olivier $\cdot$

R. S. Oosting

Department of Pharmacology, Utrecht Institute for Pharmaceutical

Sciences and Rudolf Magnus Institute of Neuroscience,

Utrecht University,

Universiteitsweg 99,

3584 CG, Utrecht, Netherlands

e-mail: t.n.douma@uu.nl

B. Olivier

Department of Psychiatry, Yale University School of Medicine,

New Haven, CT, USA

\section{Introduction}

Early-stage Alzheimer's disease (AD) is associated with marked impairments in olfactory function (Warner et al. 1986; Djordjevic et al. 2007), and neurofibrillary tangles and neuritic plaques have been found in olfactory bulbs of AD brains (Ohm and Braak 1987). In rodents, bilateral olfactory bulbectomy (OBX) leads to extensive cognitive impairments, with deficits in learning and memory (Mucignat-Caretta et al. 2006; Kelly et al. 1997). These behavioral deteriorations become apparent about 2 weeks after ablation of the olfactory bulbs (Mucignat-Caretta et al. 2006), indicating an effect beyond the sensory deficit. Indeed, it is established that anosmia does not account for the major OBX-induced changes in cognitive and other behaviors (Cain 1974; Van Riezen et al. 1977). Anatomically, neurons of the main and accessory olfactory bulbs project to cortical, amygdala, and hippocampal regions. The bulbectomy-induced behavioral deficits are probably attributable to retrograde degeneration of these neurons (Kelly et al. 1997; Song and Leonard 2005). Moreover, neuronal damage following OBX is thought to be enhanced by accompanying inflammatory reactions (Myint et al. 2007; Song et al. 2009).

One of the most robust behavioral changes induced by bulbectomy is locomotor hyperactivity in the open field test, which could be normalized by chronic, but not acute, administration of antidepressants (Breuer et al. 2007; Kelly et al. 1997). Furthermore, OBX has been reported to decrease hippocampal neurogenesis, a putative pathogenic mechanism in depression (Koo et al. 2010) that could be subsequently reversed by the antidepressant citalopram (Jaako-Movits and Zharkovsky 2006).

In addition to its role in depression, hippocampal degeneration has been suggested to be the basis, at least 
partly, for the cognitive deficits in AD (Thompson et al. 2004). Thereby, OBX was associated with increased levels of beta-amyloid protein in neocortex and hippocampus (Aleksandrova et al. 2004). Notably, drugs approved for the treatment of $\mathrm{AD}$ were reported to alleviate cognitive impairments induced by OBX, providing the model pharmacological validation with respect to $\mathrm{AD}$. In particular, both cholinesterase inhibitors (Hozumi et al. 2003; Yamamoto et al. 2010) and the NMDA-receptor antagonist memantine (Borre et al. 2010) improved cognition in OBX rats. Thus, the OBX model may also be useful for modeling (early) Alzheimer's disease.

Up till now, no drug has been shown to completely protect neurons from degradation in Alzheimer's disease. Statins or HMG-CoA reductase inhibitors were originally designed to lower cholesterol, but may also have neuroprotective and anti-inflammatory effects, and could be beneficial for AD pathology (Kojro et al. 2001; Ostrowski et al. 2007; Youssef et al. 2002). Thereby, statins could have cognition-enhancing properties, as the lipophilic drug simvastatin improved learning and memory in animal models for $\mathrm{AD}$ and traumatic brain injury, respectively ( $\mathrm{Li}$ et al. 2006; Wu et al. 2008), and enhanced long-term potentiation in hippocampal slices (Mans et al. 2010). In addition, simvastatin stimulated hippocampal neurogenesis and expression levels of the neurotrophic factors brainderived neurothropic factor (BDNF) and vascular endothelial growth factor (VEGF) (Wu et al. 2008). Furthermore, according to several epidemiological studies, statins may reduce the risk of developing AD (Darvesh et al. 2004; Sparks et al. 2005; Wolozin et al. 2000). Though, a more recent meta-analysis (Zhou et al. 2007) found no preventative effects of statins. However, in this study, no difference was made between statins that pass the bloodbrain barrier and those that do not.

In the present study, we investigated the effects of simvastatin treatment on learning and memory in OBX and control rats. Simvastatin was administered chronically (25 days), starting $48 \mathrm{~h}$ after bulbectomy, and followed by several behavioral tests (i.e., open field, passive avoidance, and two different object-place recognition tests).

\section{Materials and methods}

\section{Animals}

In total, 52 male Sprague-Dawley rats (Harlan, Zeist, The Netherlands) were used in the experiments, weighing approximately $330 \mathrm{~g}$ at the time of surgery. During surgery, three animals died. Subjects were socially housed (three or four per cage, either OBX or control animals), on a 12:12 light/dark cycle (lights on: 0600-1800 hours). Food and water were available ad libitum, room temperature was 22 $24^{\circ} \mathrm{C}$, and humidity between $30 \%$ and $60 \%$. Animals were allowed to acclimate to their surroundings for 2 weeks prior to testing and handled daily except for the weekends. At the end of the experiment, the animals were killed by decapitation, and olfactory bulb ablation was verified. Experiments were performed according to the Guide for Care and Use of Laboratory Animals and were approved by the Ethical Committee for Animal Research of Utrecht University.

\section{Olfactory bulbectomy}

The surgical procedure was performed as previously described (Breuer et al. 2007). Briefly, animals were anesthetized (isofluorane 3-4\%) and subsequently placed in a stereotaxic instrument. Two burr holes were drilled on either side of the skull, $2 \mathrm{~mm}$ in diameter. Tissue was removed with a blunt hypodermic needle and a vacuum pump. When all animals were awake and moving, they were returned to their home cages and monitored closely for 2 days. Subjects were randomly assigned to surgery or control groups. No sham operations were performed, since in previous experiments we have found that sham-operated animals do not differ from non-operated control animals (unpublished finding).

\section{Experimental design}

Upon arrival, 52 rats were randomly divided into four groups $(n=13)$. Both OBX and control animals were orally (p.o.) treated with either simvastatin $(10 \mathrm{mg} / \mathrm{kg}$, suspended in a vehicle containing $0.5 \%$ gelatin and $5 \%$ mannitol) or vehicle only. Injections were applied daily between 9 and 11 a.m. for 25 days, starting $48 \mathrm{~h}$ after surgery. Behavioral tests included the open field test (consecutively performed pre-surgically, after 2 weeks of treatment, and 1 week posttreatment), passive avoidance (habituation trials, 3 days pre-surgery; acquisition trials, 2 days pre-surgery; retention trials, 1 day pre-surgery, and 3 weeks post-surgery), and object-place recognition tests (object-place and object-inplace tests at treatment day 25 and 1 day post-treatment, respectively, following habituation sessions on treatment days 22-24). Each animal was tested in all paradigms (Fig. 1).

\section{Behavioral testing}

\section{Open field}

In an open-topped arena with light gray walls $(72 \times 72 \times$ $45 \mathrm{~cm}$ ), locomotion was videotaped for $5 \mathrm{~min}$. Luminance 


\begin{tabular}{|c|c|c|c|c|c|}
\hline-8 & $\begin{array}{llllll}-3 & -2 & -1 & 1 & 3\end{array}$ & 17 & 22 & 2425262728 & 31 \\
\hline$i$ & $\wedge \wedge$ & $\Delta$ & $\Delta$ & $\Delta \Delta \Delta \Delta \Delta$ & D \\
\hline
\end{tabular}

\begin{tabular}{|ll|}
\hline \multicolumn{2}{|l|}{ Legend } \\
OF & Open field test \\
PAH & Passive avoidance habituation \\
PAS & Passive avoidance shock \\
PAR & Passive avoidance retention \\
OBX & Olfactory bulbectomy surgery \\
HO & Habituation object tests \\
OL & Object-location task \\
OIP & Object-in-place task \\
- & Drug treatment \\
\hline
\end{tabular}

Fig. 1 Outline of all behavioral tests and interventions performed in chronological order (the day of OBX surgery is day 1). For test procedures, see text. Each animal was tested in all paradigms

was 20 lux at floor level. Distance moved was analyzed using Ethovision (Noldus, The Netherlands).

\section{Passive avoidance}

To study passive avoidance behavior, a one-trial, stepthrough passive avoidance paradigm was used (adapted from Van Riezen and Leonard 1990), where rats learn to remain in an aversive environment in order to avoid receiving a footshock. The test apparatus $(49 \times 23 \times 35 \mathrm{~cm}$, $1 \times \mathrm{w} \times \mathrm{h})$ consisted of a white, brightly lit (230 lux) compartment $(19 \times 23 \times 35 \mathrm{~cm})$ and a black, dark compartment $(29 \times 23 \times 35 \mathrm{~cm})$; connected by a sliding door $(8 \times$ $6 \mathrm{~cm}$ ). Rats were habituated to the apparatus and test procedure 1 day prior to the acquisition trial. In the habituation session, the subject was placed in the white compartment and after $10 \mathrm{~s}$, the door to the black compartment was opened. After the animal stepped inside, the door was closed and the subject was left in the black compartment for $10 \mathrm{~s}$ without a shock being delivered. During the acquisition trial, the animal was placed in the white compartment, and $10 \mathrm{~s}$ later the door was opened. As soon as the animal entered the black compartment, the door was closed and a footshock of $0.6 \mathrm{~mA}$ was presented for $3 \mathrm{~s}$, after which the animal was immediately returned to its home cage. One day after the acquisition trial (and one day before OBX surgery), the first retention trial was performed, followed by a second retention trial 21 days postsurgery. The latency time was defined as the time between opening of the door and entrance of the animal into the black compartment with all four paws. A cut-off time of $300 \mathrm{~s}$ was used. The animal was immediately taken out of the apparatus and returned to its home cage after entering the black compartment. No shock was delivered during retention trials. Any subject that showed latencies shorter than $60 \mathrm{~s}$ in the first retention trial was excluded from analysis.

\section{Object-place recognition}

In this paradigm, the ability of rats to detect a mismatch between a remembered and novel location of a familiar object is evaluated (Dix and Aggleton 1999; Barker et al. 2007). Prior to the first test, animals were habituated to an open-topped, rectangular arena $(72 \times 37.5 \times 45 \mathrm{~cm})$ with objects different from those used in test trials, for 15 $20 \mathrm{~min}$ daily for three consecutive days. The procedure of behavioral tasks consisted of an acquisition phase ( $3 \mathrm{~min}$ for object-location task and 5 min for object-in-place task), followed by a retention interval $(5 \mathrm{~min})$ and preference test phase $(3 \mathrm{~min})$. Both objects and arena were thoroughly cleaned with alcohol $(70 \% v / v)$ after each sample and test phase, in order to eliminate olfactory cues. Objects were approximately $5 \times 5 \times 5 \mathrm{~cm}$ and made of ceramics, glass, or plastic. Any animal that failed to reach a minimum of $5 \mathrm{~s}$ of object exploration in the test phase was excluded from the analysis.

\section{The object-location task}

This task assesses the rat's ability to discriminate between objects on familiar and novel locations. In the sample phase, the animal was exposed to two objects placed in the corners of the arena, $15 \mathrm{~cm}$ from the two nearest walls. During the retention phase, one of the objects was left in the original position while the other object was moved to a novel location (i.e., near the corner adjacent to the original position). The position of the displaced object (i.e., left or right) was counterbalanced within and between groups.

\section{The object-in-place task}

In this topological task, the animal's ability to recognize spatial relationships between objects is evaluated. During the sample phase, the animals were exposed to four different objects, each placed near another corner in the arena. Subsequently, the animals were allowed to explore the objects for $5 \mathrm{~min}$. In the test phase, two objects were swapped. The position of the moved objects was counterbalanced within and between groups.

\section{Discrimination ratio}

For both tasks, the discrimination ratio was calculated as time spent exploring object(s) that changed position compared to object(s) that remained in the same position relative to the total time spent exploring all objects, 
according to the formula: ( $t$ [novel] $-t$ [constant]) / ( $t$ [novel] $+t$ [constant]). Exploratory behavior was defined as orienting the nose towards the object at a distance $<4 \mathrm{~cm}$. In behavioral scoring, the experimenter was blind to the surgical and treatment status of the animal. Discrimination performance during the first minute of testing is used for analysis, as this has proven to be the most sensitive period of object recognition (Dix and Aggleton 1999). Any animal that failed to reach a minimum of $5 \mathrm{~s}$ of object exploration in the test phase was excluded from analysis.

\section{Statistical analysis}

Total distances moved in the open field test were analyzed using two-way ANOVA, followed by post hoc $t$ tests with Bonferonni correction. Assumptions of normality and homogeneity of variance were tested with KolmogorovSmirnov and Levene's tests, respectively. Passive avoidance latency times were analyzed using nonparametric statistics (Kruskal-Wallis), followed by post hoc Dun's multiple comparison test and Mann-Whitney $U$ test. Group comparisons in object recognition tests were made by twoway ANOVAs, and to discriminate if group performance was significantly above chance level, one-sample $t$ tests were used. All differences were considered to be significant if $p \leq 0.05$. Statistical calculations were carried out using SPSS 18.

\section{Results}

\section{Open field}

OBX animals were significantly more active compared to controls (two-way ANOVA, $F[1,45]=33.3, p<0.001$; post hoc corrected independent samples $t$ tests, $t[23]=-5.271, p=$ $0.000, t[22]=-3.153, p=0.01$, for vehicle and simvastatintreated animals, respectively). Simvastatin did not affect locomotion after 14 days of treatment $(F[1,45]=0.352$, ns) (Fig. 2). Two days post-treatment, a second open field test was performed giving similar outcomes (data not shown).

\section{Learning and memory}

Passive avoidance Pretreatment and pre-surgery, mean latency times to enter the black compartment did not differ between groups (not shown). Twenty-one days following surgery (and 18 days of simvastatin treatment), passive avoidance memory differed significantly between groups [Kruskal-Wallis, $H(3)=26.49, p<0.0001]$. Post hoc analysis revealed that for the two treatment conditions, OBX groups were significantly different from controls $(p<0.01)$, and that there was no significant effect of treatment.

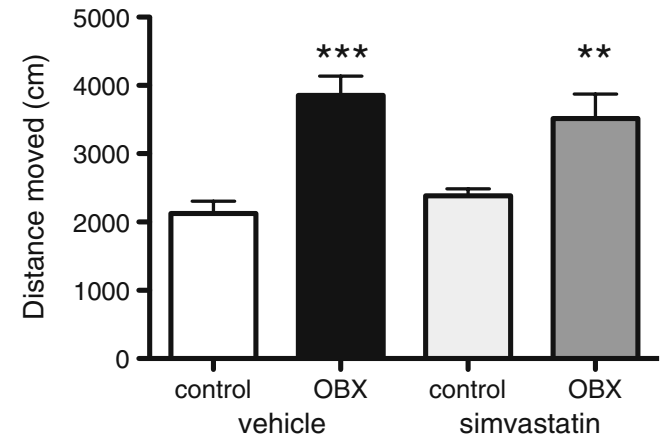

Fig. 2 Simvastatin did not affect OBX-induced hyperactivity. Rats received daily either simvastatin $(10 \mathrm{mg} / \mathrm{kg}$, p.o.) or vehicle for 14 days, starting $48 \mathrm{~h}$ post-surgery. Shown is mean+SEM of distance traveled during the first $5 \mathrm{~min}$ of the open field test. Post hoc comparisons vs. controls, ${ }^{* * *} p \leq 0.001, * * p \leq 0.01 . N=11-13$

However, in measuring latency times, we used a cut-off time of $300 \mathrm{~s}$. This highest possible value was reached by seven out of nine simvastatin-treated control animals, while only two out of ten vehicle-treated controls showed a latency time of $300 \mathrm{~s}$ (Fig. 3). Also, a single comparison of simvastatin- vs. vehicle-treated controls revealed a significant difference between the groups (Mann-Whitney, $U=22.0, p=0.047$ ).

\section{Object-place recognition}

Object-location task Twenty-seven days after bulbectomy, object-location recognition was significantly impaired in OBX rats (two-way ANOVA, $F[1,41]=12.421, p=0.001$ ) and not affected by simvastatin in any group $(F[1,41]=$ $0.025, \mathrm{~ns})$. Both control groups performed significantly better than chance (i.e., a level of zero) (one sample $t$ test, $t$ $[10]=5.867, p=0.000$ and $t[11]=6.787, p=0.000$ for statin

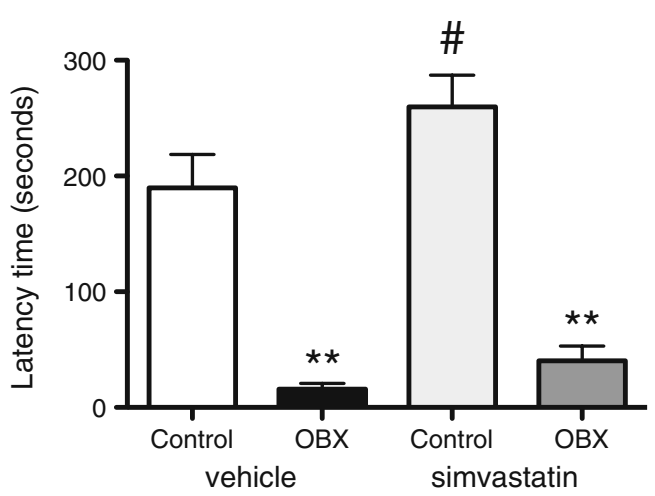

Fig. 3 Passive avoidance behavior was significantly impaired following OBX and improved by simvastatin in control animals only. Shown is the mean + SEM of the latency time to enter the dark compartment. Simvastatin $(10 \mathrm{mg} / \mathrm{kg}$, p.o.) was administered daily for 18 days. Post hoc comparisons vs. control groups, $* * p \leq 0.01$; comparison vs. vehicle treated control, \# $p<0.05$. $N=9-10$ 
and vehicle groups, respectively), whereas OBX animals did not perform above chance (Fig. 4a).

Object-in-place task Twenty-eight days after surgery, object-in-place recognition of OBX rats was significantly inferior compared to controls $(F[1,41]=5.313, p=0.026)$, which was not significantly altered by statin treatment $(F$ $[1,41]=1.262$, ns). However, additional analysis revealed that only the simvastatin-treated control group performed significantly above chance level $(t[9]=5.77, p<0.001)$ (Fig. 4b).

\section{Discussion}

The present study shows that chronic treatment with simvastatin (25 days) improves cognitive performance in control rats, as observed in object-in-place recognition and passive avoidance tasks. Furthermore, simvastatin did not improve OBX-induced behavioral deficits (i.e., object-
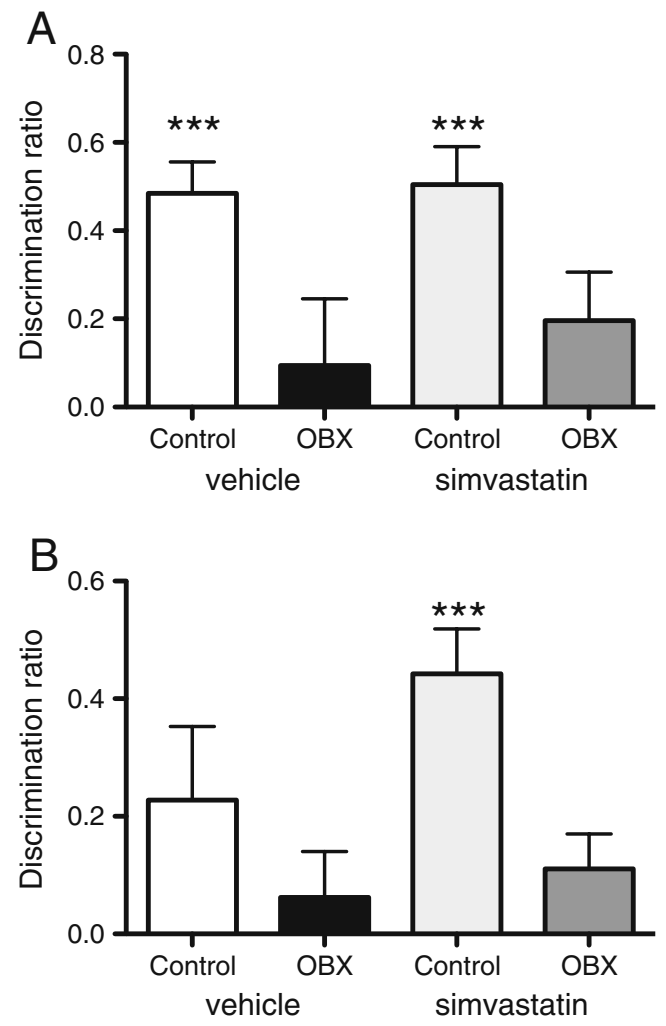

Fig. 4 Simvastatin significantly improved object-place recognition selectively for control animals. a Performance in object-location task for control and OBX groups receiving either vehicle or simvastatin. $N=$ 10-11. b Performance for each group in object-in-place task. $N=10-12$. Simvastatin $(10 \mathrm{mg} / \mathrm{kg}$, p.o.) was administered daily for 25 days. Shown is the mean+SEM of the discrimination ratio in the first minute of the test phase. $* * *$ Denotes significant difference from chance $(=0)$ (one sample $t$ test, $p \leq 0.001$ ) place recognition, open field, and passive avoidance behavior). Studies in rat and mouse models of Alzheimer's disease (Li et al. 2006) and traumatic brain injury (Wu et al. 2008) have shown that statins have neuro-restorative and anti-inflammatory properties. This raises the question as to why simvastatin proved to be ineffective in ameliorating learning and memory in the OBX rat.

One suggestion is that the brain damage resulting from ablation of the olfactory bulbs is too severe or extensive to be counteracted by simvastatin treatment. In a study by Skelin et al. (2008), it was shown that OBX leads to reduced glucose utilization in 13 different brain areas, which is indicative for a widespread reduction in brain activity. Affected brain areas included the ventral hippocampus and the amygdala. In the abovementioned traumatic brain injury study, which reported improved cognitive performance following chronic simvastatin treatment $(\mathrm{Wu}$ et al. 2008), the tissue damage was more restricted. Specifically, neuronal cell death primarily occurred in the boundary zone of the injured cortical region and the CA3 region of the hippocampus, which gave rise to spatial learning deficits.

Alternative explanations why simvastatin might have been ineffective in the OBX model could relate to dosage and time factors. In the current study, simvastatin was applied in a dose of $10 \mathrm{mg} / \mathrm{kg}$. Elsewhere, doses ranging from 1 to $50 \mathrm{mg} / \mathrm{kg}$ of simvastatin have been successfully used (Lu et al. 2007; Wu et al. 2008, 2009). Therefore, it is unlikely that our dose of $10 \mathrm{mg} / \mathrm{kg}$ was too low. Moreover, the $10 \mathrm{mg} / \mathrm{kg}$ dose was effective in the control animals. Since we started simvastatin treatment $48 \mathrm{~h}$ after OBX surgery, it is possible that at this stage, part of the neurons in brain areas beyond the olfactory bulbs had already been damaged. Our previous telemetry studies demonstrated that rat home-cage hyperactivity becomes apparent 2-3 days after OBX surgery (Vinkers et al. 2009). Furthermore, OBX-induced passive avoidance deficits have been reported to develop between $24 \mathrm{~h}$ and 14 days after surgery in mice (Hozumi et al. 2003). Taken together, it is likely that although some brain tissue may have been irreversibly damaged by the onset of treatment, there may still have been room for improvement, e.g., limiting further loss of neuronal function or even enhancing the function of remaining neurons.

Based on a reversal of locomotor hyperactivity in the open field test, the OBX model has been used to predict known antidepressant drugs across laboratories (Breuer et al. 2007; Kelly et al. 1997). In the present study, chronic simvastatin treatment did not normalize the hyperactivity response of OBX rats (Fig. 2). This was an unexpected finding, as like antidepressants (Sahay and Hen 2007), simvastatin stimulates neurogenesis (Wu et al. 2008) and the production of the neurotrophic factors BDNF and 
VEGF in the hippocampus (Wu et al. 2008). Stimulation of neurogenesis is considered necessary for the antidepressant effects in mice (Santarelli et al. 2003). In addition, chronic treatment with the anti-inflammatory drug celecoxib normalized OBX-induced hyperactivity in rats (Myint et al. 2007; Song et al. 2009). Based on the studies supporting anti-inflammatory properties of statins, we hypothesized that simvastatin would do the same. On the other hand, to the best of our knowledge, no antidepressant effects of statins have been reported in humans so far.

Although we did not find any effect of simvastatin in OBX rats, the drug enhanced behavioral performance in controls. In the beginning of the experiment, all animals went through a passive avoidance acquisition trial, in which they received a shock $(0.6 \mathrm{~mA}, 3 \mathrm{~s})$. Theoretically, this could have interfered with later task performance, for instance by increasing anxiety. This may be in particular true for the control group, since OBX animals do not recall memories acquired before surgery (see Fig. 3). The remaining question is whether the effects of simvastatin in control animals can be ascribed to enhanced cognition, or are just the result of decreased anxiety states.

In one study, using the same dose of simvastatin as we did, anxiolytic effects of the drug were reported in rats (Wang et al. 2009). Based on the present findings, it cannot be excluded that possible anxiolytic effects of simvastatin might have played a role in improving performance in the object-in-place task. However, the possible contribution of this effect is considered small, since the drug also increased latency times in the passive avoidance test-a procedure widely used in memory research (Gold 1986). Furthermore, a number of studies report statin-induced improved cognition in control mice (Li et al. 2006), possibly by modulation of signaling pathways implicated in synaptic plasticity and (spatial) memory formation (Laufs et al. 1998; Vaughan 2003). Notably, increased levels of NMDA receptors following chronic simvastatin treatment have been reported in rats (Wang et al. 2009). NMDA receptors play an important role in learning and memory (Magnusson and Cotman 1993).

All tests performed in this study are considered to be dependent on the hippocampus, though depending on the task, cortical brain areas may be involved as well (Barker et al. 2007; Burwell et al. 2004). Both, the object-in-place recognition and passive avoidance tests - where simvastatin was found to exert beneficial effects - are more complex tasks. In the passive avoidance task, contextual fear learning is evaluated, i.e., the animal has to make an association between the context and an aversive stimulus. Burwell et al. (2004) reported that passive avoidance learning requires an intact corticohippocampal circuit (i.e., hippocampus and perirhinal, postrhinal, and enthorhinal cortices). Also, in a lesion study by Barker et al. (2007), it was shown that the object-in-place task is dependent on an intact medial prefrontal and perirhinal cortex, while the object-location task was not affected by lesions in these areas.

Based on these findings, it can be hypothesized that simvastatin exerts cognition-enhancing effects in tasks that require cortical participation. On the other hand, it is also reported that simvastatin improves spatial learning in the Morris water maze ( $\mathrm{Li}$ et al. 2006), which is thought to depend on an intact hippocampus (Burwell et al. 2004; Moses et al. 2005). However, as suggested by Burwell et al. (2004), the complexity of spatial cues present during the Morris water maze test may determine whether cortical regions become involved or not. Thus, mechanisms underlying cognition-enhancing effects of simvastatin remain to be determined. Unraveling those mechanisms may be of particular importance, as in non-demented elderly, statins have been reported to improve cognition (Parale et al. 2006) and to reduce the rate of cognitive decline (Bernick et al. 2005; Sparks et al. 2010).

In conclusion, we show here that simvastatin treatment has beneficial effects on higher-order learning and memory in intact rats, but is without any (behavioral) effect in OBX rats. Based on the present findings, we present two suggestions which could explain why simvastatin was ineffective in restoring behavioral deficits induced by bulbectomy. First, it may be that the OBX-induced neuronal damage was too severe to be counteracted by simvastatin treatment. Second, simvastatin may only improve higher-order cognition that requires cortical participation, while in OBX animals (task-relevant), subcortical brain areas are also affected. Finally, the finding that simvastatin improves learning and memory in control rats, supports the view that statins may prevent or improve (early) age-associated cognitive decline in elderly humans.

Acknowledgments This study was supported in part by the Internationale Stichting Alzheimer Onderzoek (ISAO). The authors have no financial relation with the ISAO. Performed experiments comply with the current laws of the Netherlands.

Open Access This article is distributed under the terms of the Creative Commons Attribution Noncommercial License which permits any noncommercial use, distribution, and reproduction in any medium, provided the original author(s) and source are credited.

\section{References}

Aleksandrova I, Kuyichkin V, Kashparov I, Medvinskaya N, Nesterova I et al (2004) Increased level of beta-amyloid in the brain of bulbectomized mice. Biochemistry (Mosc) 69:176-180

Barker G, Bird F, Alexander V, Warburton E (2007) Recognition memory for objects, place, and temporal order: a disconnection 
analysis of the role of the medial prefrontal cortex and perirhinal cortex. J Neurosci 27:2948-2957

Bernick C, Katz R, Smith N, Rapp S, Bhadelia R, Carlson M et al (2005) Statins and cognitive function in the elderly: the cardiovascular health study. Neurology 65:1388-1394

Borre Y, Bosman E, Hendriksen H et al. (2010) Chronic memantine treatment restores passive avoidance but not spatial memory in rat following olfactory bulbectomy. FENS Abstr. vol 5, 057

Breuer M, Groenink L, Oosting R, Westenberg G, Olivier B (2007) Long-term behavioral changes after cessation of chronic antidepressant treatment in olfactory bulbectomized rats. Biol Psychiatry 61:990-995

Burwell R, Saddoris M, Bucci D, Wiig K (2004) Corticohippocampal contributions to spatial and contextual learning. J Neurosci 24:3826-3836

Cain D (1974) The role of the olfactory bulb in limbic mechanisms. Psychol Bull 81:654-671

Darvesh S, Martin E, Walsh R, Rockwood K (2004) Differential effects of lipid-lowering on human cholinesterases. Clin Biochem $37: 42-49$

Dix S, Aggleton J (1999) Extending the spontaneous preference test of recognition: evidence of object-location and object-context recognition. Behav Brain Res 99:191-200

Djordjevic J, Jones-Gotman M, De Sousa K, Chertkow H (2007) Olfaction in patients with mild cognitive impairment and Alzheimer's disease. Neurobiol Aging 29:693-706

Gold PE (1986) The use of avoidance training in studies of modulation of memory storage. Behav Neural Biol 46(1):87-98

Hozumi S, Nakagawasai O, Tan-No K, Niijima F, Yamadera F, Murata A et al (2003) Characteristics of changes in cholinergic function and impairment of learning and memory-related behavior induced by olfactory bulbectomy. Behav Brain Res 138:9-15

Jaako-Movits K, Zharkovsky A (2006) Impaired fear memory and decreased hippocampal neurogenesis following olfactory bulbectomy in rats. Eur J Neurosci 22:2871-2878

Kelly J, Wrynn A, Leonard B (1997) The olfactory bulbectomized rat as a model of depression: an update. Pharmacol Ther $74: 299-316$

Kojro E, Gimpl G, Lammich S, Marz W, Fahrenholz F (2001) Low cholesterol stimulates the nonamyloidogenic pathway by its effect on the alpha-secretase ADAM 10. Proc Natl Acad Sci USA 98:5815-5820

Koo J, Russo S, Ferguson D, Nestler E, Duman R (2010) Nuclear factor-kappaB is a critical mediator of stress-impaired neurogenesis and depressive behavior. Proc Natl Acad Sci USA 107:2669-2674

Laufs U, La Fata V, Plutzky J, Liao J (1998) Upregulation of endothelial nitric oxide synthetase by $\mathrm{HMG}$ CoA reductase inhibitors. Circulation 97:1129-1135

Li L, Cao D, Kim H, Lester R, Fukuchi K (2006) Simvastatin enhances learning and memory independent of amyloid load in mice. Ann Neurol 60:729-739

Lu D, Changsheng Q, Goussev A, Jiang H, Lu C, Schallert T (2007) Statins increase neurogenesis in the dentate gyrus, reduce delayed neuronal death in the hippocampal CA3 region, and improve spatial learning in rat after traumatic brain injury. J Neurotrauma 24:1132-1146

Magnusson R, Cotman C (1993) Effects of aging on NMDA and MK801 binding sites in mice. Brain Res 604:334-337

Mans R, Chowdhury N, Cao D, McMahon L, Li L (2010) Simvastatin enhances long-term potentiation in C57BL/6 mice. Neuroscience 166:435-444

Moses S, Cole C, Ryan J (2005) Relational memory for object identity and spatial location in rats with lesions of perirhinal cortex, amygdala and hippocampus. Brain Res Bull 65:501-512
Mucignat-Caretta C, Bondi M, Caretta A (2006) Time course of alterations after olfactory bulbectomy in mice. Physiol Behav 89:637-643

Myint A, Steinbusch H, Goeghegan L, Luchtman D, Kim Y, Leonard B (2007) Effect of the COX-2 inhibitor celecoxib on behavioural and immune changes in an olfactory bulbectomised rat model of depression. Neuroimmunomodulation 14:65-71

Ohm T, Braak H (1987) Olfactory bulb changes in Alzheimer's disease. Acta Neuropathol 73:365-369

Ostrowski S, Wilkinson B, Golde T, Landreth G (2007) Statins reduce amyloid-beta production through inhibition of protein isoprenylation. J Biol Chem 282:26832-26844

Parale G, Baheti N, Kulkarni P, Panchal N (2006) Effects of atorvastatin on higher functions. Eur J Clin Pharmacol 62:259-265

Sahay A, Hen R (2007) Adult hippocampal neurogenesis in depression. Nat Neurosci 10:1110-1115

Santarelli L, Saxe M, Gross C, Surget A, Battaglia F, Dulawa S et al (2003) Requirement of hippocampal neurogenesis for the behavioral effects of antidepressants. Science 301:805-809

Skelin I, Sato H, Diksic M (2008) Olfactory bulbectomy reduces cerebral glucose utilization: 2-[14C]deoxyglucose autoradiographic study. Brain Res Bull 79:485-492, Erratum in: Brain Res Bull (2009) 79:463

Song C, Leonard B (2005) The olfactory bulbectomised rat as a model of depression. Neurosci Biobehav Rev 29:627-647

Song C, Zhang X, Manku M (2009) Increased phospholipase A2 activity and inflammatory response but decreased nerve growth factor expression in the olfactory bulbectomized rat model of depression: effects of chronic ethyl-eicosapentaenoate treatment. J Neurosci 29:14-22

Sparks D, Sabbagh M, Connor D, Lopez J, Launer L, Browne P et al (2005) Atorvastatin for the treatment of mild to moderate Alzheimer Disease. Arch Neurol 62:753-757

Sparks D, Kryscio R, Connor D, Sabbagh M, Sparks L, Lin Y et al (2010) Cholesterol and cognitive performance in normal controls and the influence of elective statin use after conversion to mild cognitive impairments: results in a clinical trial cohort. Neurodegener Dis 7:183-186

Thompson P, Hayashi K, Zubicaray G (2004) Mapping hippocampal and ventricular change in Alzheimer disease. Neuroimage 4:1754-1766

Van Riezen H, Leonard R (1990) Effects of psychotropic drugs on the behavior and neurochemistry of olfactory bulbectomized rats. Pharmacol Ther 47:21-34

Van Riezen H, Schnieden H, Wren A (1977) Olfactory bulb ablation in the rat: behavioural changes and their reversal by antidepressant drugs. Br J Pharmacol 60(4):521-528

Vaughan C (2003) Prevention of stroke and dementia with statins: effects beyond lipid lowering. Am J Cardiol 91:23B-29B

Vinkers C, Breuer M, Westphal K, Korte S, Oosting R, Olivier B, Groenink L (2009) Olfactory bulbectomy induces rapid and stable changes in basal and stress-induced locomotor activity, heart rate and body temperature responses in the home cage. Neuroscience 159:39-46

Wang Q, Zengin A, Deng C, Li Y, Newell K, Yang G (2009) High dose of simvastatin induces hyperlocomotive and anxiolytic-like activities: the association with the up-regulation of NMDA receptor binding in the rat brain. Exp Neurol 216:132-138

Warner M, Peabody C, Flattery J, Tinklenberg J (1986) Olfactory deficits and Alzheimer's disease. Biol Psychiatry 21:116-118

Wolozin B, Kellman W, Ruosseau P, Celesia G, Siegel G (2000) Decreased prevalence of Alzheimer's disease associated with 3hydroxy-3-methylglutaryl coenzyme a reductase inhibitors. Arch Neurol 57:1439-1443

Wu H, Lu D, Jiang H, Xiong Y, Qu C, Li B et al (2008) Simvastatinmediated upregulation of VEGF and BDNF, activation of the 
$\mathrm{PI} 3 \mathrm{~K} /$ Akt pathway, and increase of neurogenesis are associated with therapeutic improvement after traumatic brain injury. J Neurotrauma 25:130-139

Wu H, Mahmood A, Dunyue L, Jiang H, Xiong Y, Zhou D (2009) Attenuation of astrogliosis and modulation of endothelial growth factor receptor in lipid rafts by simvastatin after traumatic brain injury. J Neurosurg. doi:10.3171/2009.9.JNS09859

Yamamoto Y, Shioda N, Han F, Moriguchi S, Fukunaga K (2010)

Donepezil-induced neuroprotection of acetylcholinergic neu- rons in olfactory bulbectomized mice. Yakugaku Zasshi 130:717-721

Youssef S, Stüve O, Patarroyo J, Ruiz P, Radosevich J, Mi Hur E et al (2002) The HMG-CoA reductase inhibitor, atorvastatin, promotes a Th2 bias and reverses paralysis in central nervous system autoimmune disease. Nature 420:78-84

Zhou B, Teramukai S, Fukushima M (2007) Prevention and treatment of dementia or Alzheimer's disease by statins: a meta-analysis. Dement Geriatr Cogn Disord 23:194-201 